\title{
Quality of Life Improvement after Surgery for Deep Infiltrating Endometriosis (DIE)
}

Voicu Simedrea ${ }^{1}$, Mădălin-Marius Margann ${ }^{2,3}$, Iris Cioroianu ${ }^{4}$, Raul Pătrașcu ${ }^{4}$, Andrei Mărginean ${ }^{5}$ and Roxana Nicolescu ${ }^{3}$

${ }^{1}$ Premiere Hospital Timișoara, Romania

${ }^{2}$ Department of Obstetrics and Gynecology, "Victor Babes" University of Medicine and Pharmacy, Timișoara, Romania

${ }^{3}$ Timişoara County Emergency Clinical Hospital, "Bega" University Clinic of Obstetrics and Gynecology, Timișoara, Romania

4"Victor Babeș" University of Medicine and Pharmacy, Timișoara, Romania

5"Dr. Victor Popescu” Emergency Military Clinical Hospital, Timișoara, Romania

\begin{abstract}
Endometriosis is categorized as one of the chronic benign gynecologic diseases, which causes pelvic pain and infertility, affecting almost $10 \%$ of reproductive-age women. Deeply infiltrating endometriosis (DIE) is a specific entity of endometriosis, responsible for painful symptoms, which are related to the anatomic location of the lesions. In this paper, we aim to review the current literature regarding the post-surgery quality of life improvement for DIE. Irrespective of its low sensitivity and specificity, vaginal examination and evaluation of specific symptoms should be emphasized as a basic diagnostic tool in detecting endometriosis. This will help in planning further DIE related therapeutic interventions. Out of several, transvaginal ultrasound (TVUS) has been reported as one of the widely used and excellent tools to diagnose DIE lesions in different locations (rectovaginal septum, retrocervical and paracervical areas, rectum and sigmoid and vesical wall).
\end{abstract}

Keywords: Deep infiltrating endometriosis; DIE; Quality of life; QOL; Preoperative evaluation

\section{Background}

Endometriosis is a painful and chronic gynecologic disorder, characterized by the presence of ectopic endometrium outside the endometrial cavity. Under this situation endometrial cells are implanted ectopically, that lead to retrograde menstruation via the fallopian tubes into the pelvis [1]. Endometriosis affects at least 6.3 million women and girls predominantly of reproductive age in the United States, 1 million in Canada, and millions more worldwide. It is associated with pelvic pain and infertility [2]. Peritoneal endometriosis, ovarian endometriosis and DIE are the three clinical presentations of endometriosis that have been described before [3]. Furthermore, several classifications of DIE have been proposed. In one classification, three different types of DIE are distinguished [4]:

(I) A large lesion in the peritoneal cavity, infiltrating conically with the deeper parts becoming progressively smaller is designated as type-1;

(II) In type-2, the bowel is being retracted over the lesion, and becomes deeply situated in the rectovaginal septum without infiltrating it;

(III) Spherically shaped lesions, situated deep in the rectovaginal septum, and are often only visible as a small typical lesion at laparoscopy or often not visible at all. In the year 1995, Donnez and Nisolle have proposed only two types of DIE, first being caused by the invasion of a very active peritoneal lesion deep in the retroperitoneal space. In cases of lateral peritoneal invasion, utero-sacral ligaments can be involved as well as the anterior wall of the recto-sigmoid bowel junction resulting in a retraction, adhesions and secondary obliteration of the cul-desac. A second type is pseudo-DIE where the lesion originates from the rectovaginal septum tissue and consists essentially of smooth muscle with active glandular epithelium and scanty stroma [5].

Today it is believed that endometriotic lesions can penetrate deep either into the retroperitoneal space or into the walls of the pelvic organs [6]. However, the mechanism is not clear and little is known about the impact of the different types of surgery in the treatment of DIE on complications, pain, patients' quality of life (QOL), recurrence rate and pregnancy rate or fertility. The aim of this review is therefore to evaluate the quality of life improvement after the different surgical modalities for management of DIE based on the above-mentioned parameters.

\section{Material and Method}

In this review we have searched The PUBMED (March 2005 to July 2015) for relevant articles. Heading terms "deep infiltrating endometriosis, quality of life" $(\mathrm{n} 1=33)$ and "deep infiltrating endometriosis" ( $\mathrm{n} 2=402)$ were used. All pertinent articles were retrieved without any language restriction. To ensure the relevance of the publications, additional inclusion criteria were applied. We have included only those studies that contained a clear explanation of the surgical technique, an effectual evaluation of pain and an explicit description of post-operative QOL. To ensure a complete review of the preoperative evaluation of DIE, we have also included some of the most relevant studies regarding this subject, without any restriction.

\section{Specific Symptoms of DIE}

DIE exhibits a broad spectrum of clinical manifestations. DIE can be completely asymptomatic, or can become a disorder where quality of live is heavily compromised, as DIE is defined by the presence of endometrial implants, fibrosis and muscular hyperplasia under the

*Corresponding author: Margan Mădălin-Marius, Assistant Lecturer, Department of Obstetrics and Gynecology, "Victor Babeș" University of Medicine and Pharmacy Timișoara, University Clinic of Obstetrics and Gynecology "Bega", Victor Babeș Blvd. No 12, 300226, Timișoara, Romania, Tel: +40 (0) 7262773 54; E-mail: Imarganmm@gmail.com

Received November 18, 2014; Accepted December 15, 2015; Published December 20, 2015

Citation: Simedrea M, Margan MM, Cioroianu I, Pătrașcu R, Mărginean A, et al CQuality of Life Improvement after Surgery for Deep Infiltrating Endometriosis (DIE). Journal of Surgery [Jurnalul de chirurgie]. 2015; 11(4): 137-142 DOI: 10.7438/1584-9341-11-4-4

Copyright: (c) 2015 Simedrea M, et al. This is an open-access article distributed under the terms of the Creative Commons Attribution License, which permits unrestricted use, distribution, and reproduction in any medium, provided the original author and source are credited. 
peritoneum [7]. It may also involve, in descending order of frequency, the uterosacral ligaments, the rectosigmoid colon, the vagina and the bladder. DIE may cause severe dysmenorrhoea. However, pelvic pain may be more common in women with deep, infiltrating implants postsurgery. It is reported that, DIE induced pain is due to compression or infiltration of nerves in the sub-peritoneal pelvic space by the implants [8]. However, the intensity of pain in woman with DIE correlates well with the depth and volume of infiltration [9]. DIE induced painful symptoms is very organ specific, being present in precise anatomical locations. However, multifocality is also a major characteristic of DIE Lesions [9]. DIE induced pain can thus be described as organ and location specific pain. Rectovaginal endometriosis accounts for 5\% to $10 \%$ of women with DIE [9]. It is characterized by the presence of palpable endometriotic nodules deep in the connective tissue of the pelvis. This shows profound fibrosis and fibromuscular hyperplasia [9].

\section{Surgical versus Conservative Management}

Surgery is the primary mode of treatment in most of the infiltrating diseases. Surgical treatment is very effective in relieving painful defecation, pelvic pain and dyspareunia [10]. In principle, deep endometriotic lesions should be ignored some time and should not be always treated because of its passive impact (and effect). However, intestinal and ureteral foci that cause progressive stenosis constitute indisputable reasons for operating. Otherwise, it is not necessary to opt for asymptomatic DIE surgery and should not be considered mandatory in all cases. In case there is no response to medical therapies or there is a symptom, which requires urgent surgical procedure, surgery should be the primary mode of DIE treatment. In addition, the location of the DIE lesions must dictate the choice of operating technique. Since endometriosis is located primarily on the pelvic organs, laparoscopy should be the preferred technique for diagnosis, especially in multifocal cases. It is ideal to obtain consent for surgical resection of DIE, if surgery is performed for diagnosis.

Normally, the mean number of lesions is significantly correlated with the location of the main lesion, as the percentage of isolated DIE lesions that is located on a single, varies between 29 and $83 \%$ [10]. During speculum observation, a bluish lesion is observed which is normally located in the upper third corner of posterior vaginal wall. These are normally pathognomonic for DIE diagnosis. Under such situation, a detailed medical investigation in the posterior vaginal fornix should be made for irregular appearance or for a stiff and thickened area. During this process, existence of a nodule must be sought during the vaginal touch. Even though, most clinicians sought for nodular lesion (a most standard form observed), it is not a must-to-follow rule $[11,12]$. It is also true that the outcome of clinical examination varies per the physical location of the lesion. However, signs like lateral deviation of the cervix [13] or asymmetry of the uterosacral ligaments instead of a nodule are also evident $[14,15]$. With a close examination with the speculum that finds lesions evocative of endometriosis, the upper third of the posterior surface of the vagina is infiltrated. Nevertheless, this proportion is significantly far lower in cases where the bowel or uterosacral ligaments are involved [11]. On the same note, lesions affecting vagina results in more frequent palpation of a nodule or painful infiltration during the vaginal touch [11]. So, the surgical practice must systematically include a rectal touch.

\section{Preoperative Evaluation}

Pelvic exam still represents an important step in the initial evaluation of DIE, as it offers the first perspective of preoperative assessment. Studies have demonstrated that results vary depending on DIE involvement, having low sensitivity and specificity, especially when referring to multiple localizations: ovaries, bladder, rectum, ureters etc. Fauconnier et al. in a study on 255 women evaluated the correlation between specific symptoms such as: dysmenorrhea, dyspareunia, dyschezia, gastrointestinal symptoms, and noncyclical pelvic pain and the anatomic locations of deep infiltrating endometriosis. They found a direct correlation between symptoms and location of DIE, as painful defecation was associated with vagina involvement and dyspareunia with uterosacral ligament localization. Other manifestations such as: GI symptomatology and lower urinary tract symptoms were correlated with bowel, respectively with bladder extension. It was also noticed that severe dysmenorrhea was more frequent in patients with adhesions in the Douglas pouch [16]. In a study by Chapron et al., 300 women were evaluated in order to assess the type and severity of pain symptoms and correlate them with intraoperatory findings. Endometrioma associated to severe pelvic pain strongly correlates with the presence of DIE, thus making preoperative extensive evaluation of endometriotic lesions mandatory for planning the surgical intraoperative strategy [17]. As a consequence, it is crucial to conduct additional investigations in order to map out DIE lesions accurately before surgery. The best time to conduct additional investigation is either during menstruation or just before/after the menstruation. Pelvic exam should always be associated with transvaginal ultrasound (TVUS), which is definitely the most important and widely available tool in evaluating endometriosis. Existing preliminary results need to be confirmed, but is a common view that TVUS should be conducted systematically at first intention [18]. Transrectal ultrasonography (TRUS) is one of the most reliable and widely accepted methods of diagnosing for infiltration of the bowel wall $[15,19-22]$. Infiltration of the bowel wall is an essential point of preoperative investigation as it affects the way the surgery is performed. One must consider following factors while opting for TRUS: (1) preexistence of rectal bleeding (2) possible bowel infiltration (3) possibilities of painful menstrual bowel functional symptoms in the absence of rectal bleeding and (4) possibility of a large posterior lesion. Among other methods, few clinicians also adopt cystoscopy and ureteroscopy in order to determine urinary tract mucosal infiltration, especially when bladder endometriosis is suspected [23-25]. This method allows the position of the lesion relative to the ureteral meatuses to be established. This is an important factor to consider especially when deciding on the surgical technique.

Regarding preoperative staging, the revised American Society for Reproductive Medicine (rASRM) score is the most widely used classification of endometriosis. The Enzian classification, also revised in 2011 and mainly used in the German-speaking countries, was developed as a supplement to the rASRM score, in order to provide a morphologically descriptive classification of deeply infiltrating endometriosis [26].

Magnetic resonance imaging (MRI) is another means of preoperative evaluation that provides complete and simultaneous descriptions of the anterior and posterior compartments of the pelvis [27]. Complete and simultaneous preoperative evaluation is important, as DIE lesions are most often located in the posterior compartment of the pelvis $[10,28]$. Posterior compartment of the pelvis is an area that transvaginal ultrasonography does not explore well. One drawback of MRI is that it is less sensitive for the diagnosis of bowel infiltration $[29,30]$.

Virtual modified colonoscopy is a single investigation that can visualize all affected organ systems in the pelvis and abdomen (multifocal bowel lesions, urinary tract lesions, reproductive organ lesions, and distant organ lesions such as liver involvement). The LSD/ MURO Scale is a new preoperative classification designed specifically for this method in an attempt to quantify the severity of rectogenital disease and disseminated endometriosis [31]. Further studies are essential to establish novel way of diagnosing bowel infiltration and to define the respective places of the various additional means of investigation. If the intestinal infiltration is known or suspected to exist, the bowel must be prepared preoperatively. 
In conclusion, TVUS / TRUS provide accurate information about the presence and extent of the lesions. Moreover, TVUS with or without prior preparation of the colon, can be alone an accurate non-invasive method for preoperative detection of deep-infiltrating endometriosis (DIE) involving the rectosigmoid. Ultrasound evaluation of DIE lesions is very similar to that performed laparoscopically in concordance with Enzian classification. However, involvement of the USL, pelvic and vaginal wall is difficult to assess by ultrasound only. MRI proves its worth in the correct classification of doubtful cases, in case of extrapelvic localization and abdominal adhesions. Both ultrasound and MRI are accurate diagnostic methods, with no differences in terms of disease staging. Transvaginal ultrasound is the first choice investigation if clinical suspicion is present and for rectovaginal septum evaluation. Enzian classification and rASRM score supplement each other in terms of morphological description and have common potential for classifying endometriosis. Preoperative staging permits the creation of a treatment plan and provides an early stage prognosis [26,32-35].

\section{Choosing the Best Surgical Approach}

The overall goal behind designing the best surgical approach is to achieve complete resection of all symptomatic DIE lesions during a one-step surgical intervention. To accomplish this, several surgical procedures must be associated. Operative laparoscopy based partial cystectomy is a standard method for bladder DIE. For vaginal DIE, numerous authors have demonstrated that operative laparoscopy using various techniques like electrosurgery, sharp dissection or laser $\mathrm{CO}_{2}$; exclusively laparoscopic procedure or laparoscopically assisted vaginal surgery is highly efficient. For DIE infiltrating the uterosacral ligaments, it has been shown that laparoscopic surgical resection is efficient.

In principle, the location of the endometriosis governs the choice of operating technique. Despite a huge number of available publications, there is no definitive answer available for a best possible surgical procedure that is recommended for women presenting with DIE. Previous studies show at least two widely used (and accepted) surgical approaches that are employed: (1) colorectal resection removing the rectal segment affected by the disease, and (2) nodule excision. Nodule excision may be performed by shaving the rectum. Alternatively, nodule excision is performed by removing the nodule along with the surrounding rectal wall. Again, the best surgical procedures to treat DIE lesions can be further divided into two concepts of surgery: conservative and radical. Conservative surgery is otherwise known as "nodulectomy" where intestinal DIE implant is resected. Nodulectomy is accomplished by rectal shaving [36-39] or mucosal skinning [40]. Rectal shaving allows an incomplete excision of microscopic implants, and lead to cyclic pain or digestive complaints. However, as cyclic pain may be controlled by post-operative hormonal treatment, they should not lend support to an argument for a more aggressive surgical approach. In case of bladder endometriosis, partial cystectomy is the surgical treatment of reference. This operation has been conducted by operative laparoscopy as described elsewhere [41,42]. Moreover, clinicians use laparoscopic surgical resection in cases of deep endometriosis infiltrating the uterosacral ligaments [43-47]. In this case, it is necessary to conduct ureterolysis to be able to execute the uterosacral ligament (USL) nodule completely without any risk of ureter injury. Decision on laparoscopic surgery is totally dependent upon the nature of USL. A bilateral surgery is conducted if a nodule affects both USLs. However, a healthy contralateral ligament should not be resected if the USL lesion is unilateral.

Approaching an advanced stage of endometriosis disease can be a real challenge, as it should take into consideration the real extent of infiltration and all possible complications that may appear. Angioni et al. demonstrated that incomplete surgery of DIE can eventually lead to higher rates of pain recurrences and even to repeated surgery accompanied by medical therapies [48].

\section{Determining Quality of Life Improvement after Surgery for DIE}

Quality of life (QOL) and health-related satisfaction of DIE patients can be assessed with the Medical Outcomes Survey Short Form 36 (MOS-SF-36). Available in several languages, MOS-SF-36 is the most widely used generic instrument to evaluate health-related quality of life and offers a simple tool to help clinicians select and inform patients who might benefit from DIE surgery [49]. Previous study shows that preoperative assessment of QOL with the SF-36 questionnaire can predict the QOL improvement after laparoscopic resection for endometriosis [50]. It is very important to emphasize patient's pain during the preoperative examination, which normally has a higher impact on the other components of QOL [51]. However, conservative surgeries in young women have a higher rate of pain recurrence [52]. Same study has demonstrated that in the absence of bowel resection in women with DIE, intestinal endometriosis is the factor most strongly associated with the actuarial recurrence rate [52].

One flip side of this questionnaire is that, SF-36 contains 36 items and thus places a considerable burden on both patients and investigators [53]. To overcome this issue, Ware and colleagues, therefore, decided to develop a substantially shorter questionnaire-the SF-12-reducing the number of items from 36 to 12 [54]. Clinicians found that the SF12 summary measures are highly correlated with the SF-36 summary measures. In addition, SF-12 items explained about maximum variation of the SF-36 summary measures. SF-12 also reproduces eightscale profile with fewer levels than SF-36 scales and yields less precise scores, as would be expected for single-item and two-item scales [50]. However, for large group of studies, confidence intervals are largely determined by sample size and hence these differences are not as important.

Previous studies have evaluated the impact of surgery on quality of life using visual analogue scale (VAS) [55,56]. From a group of patients with endometriosis, a significant negative correlation between VAS rating and quality of life has been evaluated by using MOS SF-36 questionnaire [57]. In addition, the same group has found a relation between hyperalgesia to pressure pain threshold measured and the impairment of SF-36 physical function as well as mental health parameters. All these interesting facts emphasize the systematic use of MOS SF-36 questionnaire as a tool, especially to identify patients who may have a benefit of surgery. On the same note, Abbott et al. (2004) have demonstrated a placebo effect of surgery on quality of life in $30 \%$ of patients with DIE [58]. Using qualitative and semiquantitative evaluations of symptoms, Redwine and Wright (2001) has convincingly demonstrated that women with predominant low back pain or asthenias are less likely candidates for extensive surgery [59]. However, we observed these data are partly in contrast with a previous report [60] with no reduction in medium- or long-term frequency and severity of recurrent dysmenorrhea after laparoscopic uterosacral ligament resection.

A study published by Mabrouk et.al (2011) on 100 patients who underwent laparoscopic surgery for DIE evaluated quality of life through the QOL questionnaire, the short form 36 (SF-36), which was completed preoperatively and postoperatively at 6-months. Laparoscopic excision of DIE lesions performed either by intestinal segmental resection or by nodule shaving, significantly improved the general symptomatology in DIE, with an increase in patient's general status and even psycho-emotional condition. The authors encourage clinicians to use this questionnaire when assessing women's healthrelated quality of life outcome after surgery for DIE [61]. Dae Gy Hong et al. evaluated the outcomes on health-related quality of life (HRQOL) of radical excision of DIE in Douglas cul-de-sac among 390 patients who underwent laparoscopic surgery. They evaluated the preoperative and postoperative visual analog scale (VAS) pain scores 
and HRQOL data from the 36-item Short Form (SF-36) questionnaire and concluded that radical excision of DIE is safe and is associated with significant improvement in QOL, especially in terms of pain [62]. Another study by Angioni et al. demonstrated that complete surgical excision of deep endometriosis is associated with better long-lasting improvement in quality of life. They encouraged surgeons to completely excise DIE implants when possible, as administration of GnRHa is followed by only a temporary improvement of symptomatology when incomplete surgery is performed [48]. In a study published by Ruffo et al., long-term outcome after laparoscopic bowel resections for DIE was evaluated on a number of 900 cases. Bowel resection for endometriosis is associated with an acceptable postoperative complication rate and significant improvement in symptoms (except for rectal bleeding and dysuria). Unfortunately, the median follow-up was just 54 months, which can be considered as a bias, so further studies need to be done in order to confirm the results [63].

The study published by Lukic et al. showed significant improvement in women with endometriosis and deep dyspareunia who underwent laparoscopic interventions. After a six-month follow-up, there was a significant improvement either in painful symptoms, but also in the quality of sexual and social lifes [64]. Unfortunately, there are a few studies evaluating the quality of sex life in women with dyspareunia and endometriosis before and after surgical treatment. Abbott et al. evaluated the surgical outcomes in 135 women, demonstrating improvement in dyspareunia and sexual pleasure using the Sexual Active Questionnaire (SAQ), with a 2-5 year follow-up [65]. Ferrero et al. also showed an increase in the number of coituses and more satisfactory orgasm among 68 women who underwent surgical intervention for endometriosis [66].

A pilot study on 20 patients with DIE and colorectal infiltration who benefited from osteopathic manipulative therapy, showed an improvement of the quality of life evaluated by the SF-36 questionnaire. Further randomized studies are required to correctly evaluate the benefits and outcomes of this technique [67].

\section{Identifying Patients Most Likely to Benefit from Surgery}

The latest review on the subject clearly concludes that surgery should be indicated only in the following situations: patients who present with significant dyspareunia and dyschezia that results in major impairment of quality of life (evaluated by VAS . 7), patients who present with signs of bowel obstruction, and patients who have failed previous in vitro fertilization (IVF) cycles [68].

Therefore, a thorough preoperative diagnostic investigation and careful detailed counseling are of major importance to understand which patients are most likely to benefit from DIE surgery. A good understanding of family history and physical examination of the patients helps predicting clinicians to evaluate the risks and benefits of surgery on an individual basis. Patients with prior information of the intestinal and urologic systems are the good candidates to schedule intraoperative consultation. Laparoscopic visualization remains the gold standard for diagnosis of endometriosis and preoperative imaging may also help guide therapeutic approaches, enabling patient counseling prior to surgery. Patients with pain should undergo a trial of empiric hormonal treatment, especially those who fail to benefit from laparoscopy. Patients with unsatisfactory preoperative function are most likely to improve, especially those with worse preoperative imagery. However, patients with a previous family history of DIE are less likely to gain functional improvement. Previous studies report a worse preoperative physical function as a strong predictor of functional improvement. This is true for patients who especially fall under upper quartile of the change in SF-36 function score [69]. One must consider predictive variables and operationalize into a clinical scoring tool to identify patients who are most likely to benefit from DIE surgery in terms of a clinically meaningful improvement in SF-36 function score.

\section{Conclusion}

A delay between onset of symptoms and diagnosis of DIE is mainly attributed to insensitivity of specific signs and available diagnostic tests. There is a clear need for a comprehensive preoperative evaluation of the disease with precise description of the morphologic extension. This mandatory step can provide the necessary surgical planning and predict possible future quality of life improvements. Patients should be precisely informed and counseled about treatment options and expected results.

\section{Conflict of interest}

The authors have no conflict of interest to report.

\section{References}

1. Sampson JA (1927) Peritoneal endometriosis due to menstrual dissemination of endometrial tissue into the pelvic cavity. Am J Obstet Gynecol 14: 422-469.

2. Kennedy S, Bergqvist A, Chapron C, D'Hooghe T, Dunselman G, et al. (2005) ESHRE guideline for the diagnosis and treatment of endometriosis. Hum Reprod 20:2698-2704.

3. Donnez J, Nisolle M, Grandjean P, Gillerot S, Clerckx F (1992) The place of $\mathrm{GnRH}$ agonists in the treatment of endometriosis and fibroids by advanced endoscopic techniques. Br J Obstet Gynaecol 99: 31-33.

4. Koninckx PR, Martin D (1994) Treatment of deeply infiltrating endometriosis Curr Opin Obstet Gynecol 6: 231-241.

5. Donnez J, Nisolle M (1995) Advanced laparoscopic surgery for the removal of rectovaginal septum endometriotic or adenomyotic nodules. Baillieres Clin Obstet Gynaecol 9: 769-774.

6. Cornillie FJ, Oosterlynck D, Lauweryns JM, Koninckx PR (1990) Deeply infiltrating pelvic endometriosis: histology and clinical significance. Fertil Steril 53:978-983.

7. Garry R (2004) The endometriosis syndromes: a clinical classification in the presence of aetiological confusion and therapeutic anarchy. Hum Reprod 19 760-768.

8. Fauconier A, Chapron C (2005) Endometriosis and pelvic pain: epidemiological evidence of the relationship and implications. Hum Reprod 11: 595-606.

9. Speroff L, Fritz MA (2005) Endometriosis. In: Clinical Gynecologic Endocrinology and Infertility ( $7^{\text {th }}$ edtn), Lippincott Williams \&Wilkins, PA, USA.

10. Chapron C, Fauconnier A, Vieira M, Barakat H, Dousset B, et al. (2003) Anatomical distribution of deeply infiltrating endometriosis: surgical implications and proposition for a classification. Hum Reprod 18: 157-161.

11. Chapron C, Dubuisson JB, Pansini V, Vieira M, Fauconnier A, et al. (2002) Clinical examination is not sufficient for the diagnosis and establishing the location of deeply infiltrating endometriosis. J Am Assoc Gynecol Laparosc 9: 115-119.

12. Koninckx PR, Meuleman C, Oosterlynck D, Cornillie FJ (1996) Diagnosis of deep endometriosis by clinical examination. Fertil Steril 65: 280-287.

13. Propst AM, Storti K, Barbieri RL (1998) Lateral cervical displacement is associated with endometriosis. Fertil Steril 70: 568-570.

14. Chapron C, Dubuisson JB (1996) Laparoscopic treatment of deep endometriosis located on the uterosacral ligaments. Hum Reprod 11: 868-873.

15. Camagna O, Dupuis O, Soncini E, B Martin, Palazzo L, et al. (2002) Prise en charge surgicale des nodules endométriosiques de la cloison recto-vaginale. A propos d'une série continue de 40 cas. Acta Endoscop 32:47-57.

16. Chapron C, Santulli P, de Ziegler D, Noel JC, Anaf V, et al. (2012) Ovarian endometrioma: severe pelvic pain is associated with deeply infiltrating endometriosis. Hum Reprod 27: 702-711.

17. Hudelist G, English J, Thomas AE, Tinelli A, Singer CF, et al. (2011) Diagnostic accuracy of transvaginal ultrasound for non-invasive diagnosis of bowel endometriosis: systematic review and meta-analysis. Ultrasound Obstet Gynecol, 37: 257-263.

18. Chapron C, Dumontier I, Dousset B, Fritel X, Tardif D, et al. (1998) Results and role of rectal endoscopic ultrasonography for patients with deep pelvic endometriosis. Hum Reprod 13: 2266-2270.

19. Fedele L, Bianchi S, Portuese A, Borruto F, Dorta M (1998) Transrectal ultrasonography in the assessment of rectovaginal endometriosis. Obstet Gynecol 91: 444-448. 
20. Schröder J, Löhnert M, Doniec JM, Dohrmann P (1997) Endoluminal ultrasound diagnosis and operative management of rectal endometriosis. Dis Colon Rectum 40: 614-617.

21. Abrão MS, Neme RM, Averbach M, Petta CA, Aldrighi JM (2004) Rectal endoscopic ultrasound with radial probe in the assessment of rectovaginal endometriosis. J Am Assoc Gynecol Laparosc 11: 50-54.

22. Vercellini P, Meschia M, De Giorgi O, Panazza S, Cortesi I, et al. (1996) Bladder detrusor endometriosis: clinical and pathogenetic implications. J Urol 155: 84-86.

23. Savoca G, Trombetta C, Troiano L, Guaschino S, Raber M, et al. (1996) Echographic, MRI and CT features in a case of bladder endometriosis. Arch Ital Urol Androl 68: 193-196 [Article in Italian].

24. Bazot M, Detchev R, Cortez A, Amouyal P, Uzan S, et al. (2003) Transvaginal sonography and rectal endoscopic sonography for the assessment of pelvic endometriosis: a preliminary comparison. Hum Reprod 18: 1686-1692.

25. Haas D, Shebl O, Shamiyeh A, Oppelt P (2013) The rASRM score and the Enzian classification for endometriosis: their strengths and weaknesses. Acta Obstet Gynecol Scand 92: 3-7

26. Balleyguier C, Chapron C, Dubuisson JB, Kinkel K, Fauconnier A, et al. (2002) Comparison of magnetic resonance imaging and transvaginal ultrasonography in the diagnosis of bladder endometriosis. J Am Assoc Gynecol Laparosc 9: 15-23

27. Redwine DB (1999) Ovarian endometriosis: a marker for more extensive pelvic and intestinal disease. Fertil Steril 72: 310-315.

28. Kondo W, Bourdel N, Jardon K, Tamburro S, Cavoli D, et al. (2011) Comparison between standard and reverse laparoscopic techniques for rectovaginal endometriosis. Surg Endosc 25: 2711-2717.

29. Kondo W, Bourdel N, Zomer MT, Slim K, Botchorischvili R, et al. (2013) Surgery for deep infiltrating endometriosis: Technique and rationale. Front Biosci (Elite Ed) 5: 316-332.

30. Van der Wat J, Kaplan MD (2015) Modified Virtual Colonoscopy in the Diagnosis and Quantification of Bowel and Disseminated Endometriosis. Surg Technol Int 26: 19-24.

31. Bazot M, Lafont C, Rouzier R, Roseau G, Thomassin-Naggara I, et al. (2009) Diagnostic accuracy of physical examination, transvaginal sonography, rectal endoscopic sonography, and magnetic resonance imaging to diagnose deep infiltrating endometriosis. Fertil Steril 92: 1825-1833.

32. Carbognin G, Girardi V, Pinali L, Raffaelli R, Bergamini V, et al. (2006) Assessment of pelvic endometriosis: correlation of US and MRI with laparoscopic findings. Radiol Med 111: 687-701.

33. Dietmar H, Radek C, Alwin H, Wolfgang S, Wolfgang W, et al. (2013) Preoperative planning of surgery for deeply infiltrating endometriosis using the ENZIAN classification. Eur J Obstet Gynecol Reprod Biol 166: 99-103.

34. Donnez J, Squifflet J (2010) Complications, pregnancy and recurrence in a prospective series of 500 patients operated on by the shaving technique for deep rectovaginal endometriotic nodules. Hum Reprod 25: 1949-1958.

35. Koninckx PR, Ussia A, Adamyan L, Wattiez A, Donnez J (2012) Deep endometriosis: definition, diagnosis, and treatment. Fertil Steril 98: 564-571.

36. Chapron C, Dubuisson JB (1999) Laparoscopic management of bladder endometriosis. Acta Obstet Gynecol Scand 78: 887-890.

37. Nezhat C, Nezhat F, Nezhat CH, Nasserbakht F, Rosati M, et al. (1996) Urinary tract endometriosis treated by laparoscopy. Fertil Steril 66: 920-924.

38. Chopin N, Vieira M, Borghese B, Foulot H, Dousset B, et al. (1999) Operative management of deep endometriosis infiltrating the uterosacral ligaments. J Am Assoc Gynecol Laparosc 6: 31-37.

39. Garry R, Clayton R, Hawe J (2000) The effect of endometriosis and its radical laparoscopic excision on quality of life indicators. BJOG 107: 44-54.

40. Koninckx PR, Timmermans B, Meuleman C, Penninckx F (1996) Complications of CO2-laser endoscopic excision of deep endometriosis. Hum Reprod 11: 2263-2268.

41. Redwine DB (1991) Conservative laparoscopic excision of endometriosis by sharp dissection: life table analysis of reoperation and persistent or recurrent disease. Fertil Steril 56: 628-634.

42. Chapron C, Jacob S, Dubuisson JB, Vieira M, Liaras E, et al. (2001) Laparoscopically assisted vaginal management of deep endometriosis infiltrating the rectovaginal septum. Acta Obstet Gynecol Scand 80: 349-354.

43. Jenkinson C, Coulter A, Wright L (1993) Short form 36 (SF36) health survey questionnaire: normative data for adults of working age. BMJ 306: 1437-1440.
44. Dubernard G, Rouzier R, David-Montefiore E, Bazot M, Darai E (2008) Use of the SF-36 questionnaire to predict quality-of-life improvement after laparoscopic colorectal resection for endometriosis. Hum Reprod 23: 846-851.

45. Dubernard G, Piketty M, Rouzier R, Houry S, Bazot M, et al. (2006) Quality of life after laparoscopic colorectal resection for endometriosis. Hum Reprod 21 1243-1247.

46. Angioni S, Pontis A, Dessole M, Surico D, De Cicco Nardone C, et al. (2015) Pain control and quality of life after laparoscopic en-block resection of deep infiltrating endometriosis (DIE) vs. incomplete surgical treatment with or without GnRHa administration after surgery. Arch Gynecol Obstet 291: 363-370.

47. Fedele L, Bianchi S, Zanconato G, Bettoni G, Gotsch F (2004) Long-term follow-up after conservative surgery for rectovaginal endometriosis. Am J Obstet Gynecol 190: 1020-1024.

48. Iglesias C, Torgerson D (2000) Does length of questionnaire matter? A randomised trial of response rates to a mailed questionnaire. J Health Serv Res Policy 5: 219-221.

49. Ware JE, Kosinski M, Keller SD (1998) SF-12: how to score the SF-12 physical and mental health summary scales. ( $3^{\text {rd }}$ edtn), QualityMetric Incorp, Lincoln.

50. Jones KD, Sutton C (2003) Patient satisfaction and changes in pain scores after ablative laparoscopic surgery for stage III-IV endometriosis and endometriotic cysts. Fertil Steril 79: 1086-1090.

51. Darai E, Thomassin I, Barranger E, Detchev R, Cortez A, et al. (2005) Feasibility and clinical outcome of laparoscopic colorectal resection for endometriosis. Am J Obstet Gynecol 192: 394-400.

52. Laursen BS, Bajaj P, Olesen AS, Delmar C, Arendt-Nielsen L (2005) Health related quality of life and quantitative pain measurement in females with chronic non-malignant pain. Eur J Pain 9: 267-275.

53. Abbott J, Hawe J, Hunter D, Holmes M, Finn P, et al. (2004) Laparoscopic excision of endometriosis: a randomized, placebo-controlled trial. Fertil Steril 82: 878-884

54. Redwine DB, Wright JT (2001) Laparoscopic treatment of complete obliteration of the cul-de-sac associated with endometriosis: long-term follow-up of en bloc resection. Fertil Steril 76: 358-365.

55. Vercellini P, Aimi G, Busacca M, Apolone G, Uglietti A, et al. (2003) Laparoscopic uterosacral ligament resection for dysmenorrhea associated with endometriosis: results of a randomized, controlled trial. Fertil Steril 80: 310-319.

56. MacWilliam CH, Yood MU, Verner JJ, McCarthy BD, Ward RE (1996) Patientrelated risk factors that predict poor outcome after total hip replacement. Health Serv Res 31: 623-638.

57. Cushnaghan J, Coggon D, Reading I, Croft P, Byng P, et al. (2007) Long-term outcome following total hip arthroplasty: a controlled longitudinal study. Arthritis Rheum 57: 1375-1380.

58. Jones CA, Voaklander DC, Johnston DW, Suarez-Almazor ME (2001) The effect of age on pain, function, and quality of life after total hip and knee arthroplasty. Arch Intern Med 161: 454-460.

59. Mabrouk M, Montanari G, Guerrini M, Villa G, Solfrini S, et al. (2011) Does laparoscopic management of deep infiltrating endometriosis improve quality of life? A prospective study. Health Qual Life Outcomes 9: 98.

60. Hong Dae Gy, Kim Joo Young, Lee Yoon Hee, Chong Gun Oh, Cho Young Lae, et al. (2014) Safety and Effect on Quality of Life of Laparoscopic Douglasectomy with Radical Excision for Deeply Infiltrating Endometriosis in the Cul-de-Sac. J Laparoendosc Adv Surg Tech A 24: 165-170.

61. Ruffo G, Scopelliti F, Manzoni A, Sartori A, Rossini R, et al. (2014) LongTerm Outcome after Laparoscopic Bowel Resections for Deep Infiltrating Endometriosis : A Single-Center Experience after 900 Cases. Biomed Res Int 2014: 463058

62. Lukic A, Di Properzio M, De Carlo S, Nobili F, Schimberni M, et al. (2015) Quality of sex life in endometriosis patients with deep dyspareunia before and after laparoscopic treatment. Arch Gynecol Obstet.

63. Abbott JA, Hawe J, Clayton RD, Garry R (2003) The effects and effectiveness of laparoscopic excision of endometriosis: a prospective study with 2-5 year follow-up. Hum Reprod 18: 1922-1927.

64. Ferrero S, Abbamonte LH, Giordano M, Ragni N, Remorgida V (2007) Deep dyspareunia and sex life after laparoscopic excision of endometriosis. Hum Reprod 22: 1142-1148.

65. Daraï C, Deboute O, Zacharopoulou C, Laas E, Canlorbe G, et al. (2015) Impact of osteopathic manipulative therapy on quality of life of patients with deep infiltrating endometriosis with colorectal involvement: results of a pilot study. Eur J Obstet Gynecol Reprod Biol 188: 70-73. 
66. Abrão MS, Petraglia F, Falcone T, Keckstein J, Osuga Y, et al. (2015) Deep endometriosis infiltrating the recto-sigmoid: critical factors to consider before management. Human Reproduction Update 21: 329-339.
67. Quintana JM, Escobar A, Aguirre U, Lafuente I, Arenaza JC (2009) Predictors of health-related quality-of-life change after total hip arthroplasty. Clin Orthop Relat Res 467: 2886-2894. 